\title{
Compressive sensing in a photonic link with optical integration
}

\author{
Chen, Ying; Yu, Xianbin; Chi, Hao; Jin, Xiaofeng; Zhang, Xianmin; Zheng, Shilie; Galili, Michael
}

Published in:

Optics Letters

Link to article, DOI:

10.1364/OL.39.002222

Publication date:

2014

Document Version

Publisher's PDF, also known as Version of record

Link back to DTU Orbit

Citation $(A P A)$ :

Chen, Y., Yu, X., Chi, H., Jin, X., Zhang, X., Zheng, S., \& Galili, M. (2014). Compressive sensing in a photonic link with optical integration. Optics Letters, 39(8), 2222-2224. https://doi.org/10.1364/OL.39.002222

\section{General rights}

Copyright and moral rights for the publications made accessible in the public portal are retained by the authors and/or other copyright owners and it is a condition of accessing publications that users recognise and abide by the legal requirements associated with these rights.

- Users may download and print one copy of any publication from the public portal for the purpose of private study or research.

- You may not further distribute the material or use it for any profit-making activity or commercial gain

- You may freely distribute the URL identifying the publication in the public portal 


\title{
Compressive sensing in a photonic link with optical integration
}

\author{
Ying Chen, ${ }^{1,2}$ Xianbin Yu, ${ }^{2}$ Hao Chi, ${ }^{1, *}$ Xiaofeng Jin, ${ }^{1}$ Xianmin Zhang, ${ }^{1}$ Shilie Zheng, ${ }^{1}$ and Michael Galili ${ }^{2}$ \\ ${ }^{1}$ Department of Information Science and Electronic Engineering, Zhejiang University, 38 Zheda Road, Hangzhou 310027, China \\ ${ }^{2}$ Department of Photonics Engineering, Technical University of Denmark, 2800 Kgs. Lyngby, Denmark \\ ${ }^{*}$ Corresponding author: chihao@zju.edu.cn
}

Received December 30, 2013; revised February 28, 2014; accepted March 8, 2014;

posted March 10, 2014 (Doc. ID 203894); published April 2, 2014

\begin{abstract}
In this Letter, we present a novel structure to realize photonics-assisted compressive sensing (CS) with optical integration. In the system, a spectrally sparse signal modulates a multiwavelength continuous-wave light and then is mixed with a random sequence in optical domain. The optical signal passes through a length of dispersive fiber, the dispersion amount of which is set to ensure that the group delay between the adjacent wavelength channels is equal to the bit duration of the applied random sequence. As a result, the detected signal is a delay-and-sum version of the randomly mixed signal, which is equivalent to the function of integration required in CS. A proof-of-concept experiment with four wavelengths, corresponding to a compression factor of 4 , is demonstrated. More simulation results are also given to show the potential of the technique. (c) 2014 Optical Society of America

OCIS codes: (320.7085) Ultrafast information processing; (250.4745) Optical processing devices.

http://dx.doi.org/10.1364/OL.39.002222
\end{abstract}

Compressive sensing (CS) is believed to be a promising technique for the acquisition of wideband sparse signals, offering the possibility for high-resolution capture of physical signals from measurements well below the number expected from the Nyquist sampling theorem. The CS process includes a linear projection from $N$ pieces of information to $M$ samples $(M \ll N)$ and a nonlinear reconstruction of the signal with $N$ pieces from $M$ samples [1]. A random demodulator (RD) and a modulated wideband converter (MWC) are two major ways to realize CS for the acquisition of a wideband sparse signal in the time domain. Realizing CS in the optical domain has attracted much interest in recent years due to the high bandwidth offered by photonic components [2-12]. The potential of photonic CS was initially demonstrated by Valley and co-workers in $[2,3]$. They proposed a method of realizing random mixing in the optical domain with a spatial light modulator (SLM), in which the random sequence is recorded on a static SLM in a pulse shaper and the RF signal is modulated on a wavelength-chirped optical pulse. The RD in a photonic link was demonstrated in [4,5]. A photonic MWC was initially proposed in [6] and recently experimentally demonstrated based on a novel effective time delay pattern in [7]. Since the MWC involves parallel channels, the photonic RD is easier to implement. CS in combination with the technique of photonic time stretching was proposed in [8] and can achieve a higher compression ratio. In the $\mathrm{RD}$, there is a random mixing stage in which the input sparse signal to be measured is mixed with a random sequence at or above the Nyquist rate of the signal, and a low-pass filter or an integrator follows the mixer. In [9], Bosworth and Foster demonstrated a photonic architecture for realizing CS in which the sparse RF signal (mixed with a random sequence) is modulated on a chirped pulse and the optical integration of the mixed product is achieved by using dispersion-based pulse compression. Single-shot detection and signal recovery for high-speed CS was experimentally demonstrated.

In this Letter, we propose a photonic CS model, in which the input RF signal to be measured is mixed with the random sequence in the optical domain and the optical integration of the mixed product is achieved by using continuous optical carriers. In our approach, a multiwavelength continuous-wave source and dispersive fiber are applied and the detected signal is a delayand-sum version of the mixed product, which is equivalent to the function of integration required in CS. Since the integration function is realized in the optical domain, the bandwidth of the photodetector (PD) can be dramatically relaxed. A proof-of-concept experiment with compression factor of 4 is successfully demonstrated. We also give more simulation results to show the potential of the given approach.

The schematic illustration of the proposed photonic CS is shown in Fig. 1(a). In the system, the incoming spectrally sparse signal is modulated on a multiwavelength optical carrier via a Mach-Zehnder modulator (MZM).
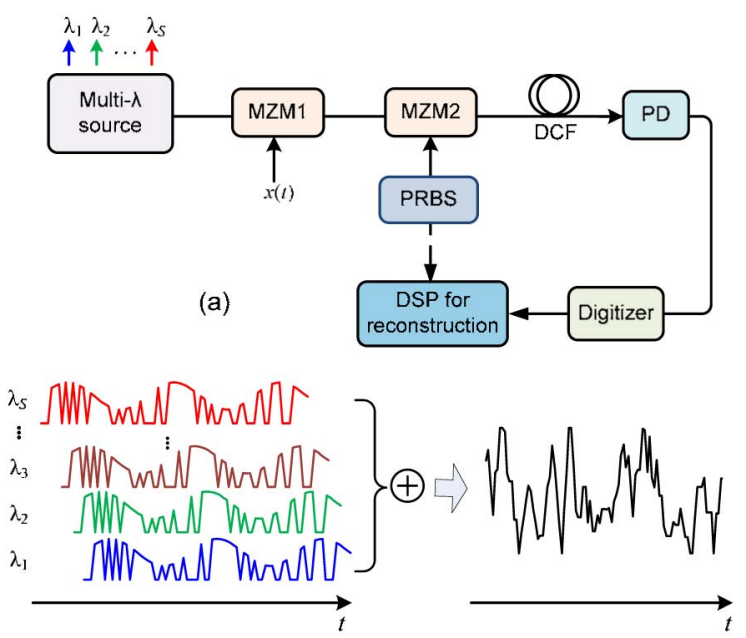

(b)

Fig. 1. (a) Schematic illustration of the proposed photonic CS structure and (b) the principle of optical integration (MZM, Mach-Zehnder modulator; PRBS, pseudo-random bit sequence; DCF, dispersion-compensation fiber; PD, photodetector; DSP, digital signal processing). 
The modulated signal is mixed with a pseudo-random bit sequence (PRBS) via another MZM. The mixed signal passes through a length of dispersion-compensation fiber (DCF) to generate group delay among the multiwavelength channels. As the multiwavelength source is incoherent, the detected signal is the sum of the signals in all wavelength channels. Therefore, the function of delayand-sum is realized. Note that, to realize precise optical integration, the wavelength space and the dispersion amount should be designed to ensure that the group delay between adjacent wavelengths is equal to the bit duration of the applied PRBS. The principle of optical integration is shown in Fig. 1(b). For a system with $S$ wavelengths, the values of consecutive $S$ bits of the mixed product $(\mathrm{RF} \times \mathrm{PRBS})$ are summed. The requirement for the sampling rate of the following digitizer is $1 / S$ of the speed of PRBS, which means the compression factor of the CS system is $S$. It should be noted that the bit rate of the PRBS should be at or above the Nyquist rate of the applied sparse signal.

The CS process can be modeled as $\mathbf{y}=\boldsymbol{\Phi} \mathbf{x}$, where $\mathbf{x}$ is the signal to be measured of dimension $N, \mathbf{y}$ is the measurement result of dimension $M(M<<N)$, and $\boldsymbol{\Phi}$ is an $M \times N$ pseudo-random matrix denoting the measurement process. According to the CS theory, signal reconstruction from $\mathbf{y}$ to $\mathbf{x}$ can be accomplished by solving an optimization problem given that $\mathbf{x}$ is sparse in a domain [1].

The measurement matrix $\boldsymbol{\Phi}$ is decided by the applied PRBS, the impulse response of the optical integration process, and the downsampling of the digitizer. Suppose the sparse signal to be measured is $x(t)$. Under the small signal approximation, the mixed product is $[1+a x(t)] r(t)$, where $r(t)$ is the applied PRBS and $\alpha$ is the modulation index of the signal on the optical carrier. The detected signal at the output of PD can be expressed as

$$
\sum_{k=0}^{S-1}[1+\alpha x(t-k T)] r(t-k T),
$$

where $T$ is the bit duration of the PRBS and $S$ is the wavelength number. The downsampling rate is equal to $S$. The measured signal at the output of the digitizer is as

$$
\sum_{m=0}^{M} \sum_{k=0}^{S-1}[1+\alpha x(m S T-k T)] r(m S T-k T) .
$$

The above measurement process can be modeled by a measurement matrix, in which the random sequence is modeled as $\operatorname{diag}[r(t)]$.

A proof-of-concept experiment with the setup shown in Fig. 1(a) is implemented to demonstrate the proposed photonic CS with four wavelength channels. The wavelengths are 1548.00, 1549.51, 1551.02, and $1552.52 \mathrm{~nm}$. Two MZMs (MZM1 and MZM2) with bandwidth of $10 \mathrm{GHz}$ are used for signal modulation and PRBS modulation. An erbium-doped fiber amplifier is used between the two MZMs to compensate the link loss. A coil of DCF with dispersion amount of $-1330 \mathrm{ps} / \mathrm{nm}$ at $1550 \mathrm{~nm}$ is applied. Note that the slight difference in wavelength spaces is induced by the higher order dispersion in DCF. A single-tone RF signal with frequency of $200 \mathrm{MHz}$ is employed as the signal to be measured. The input signal in the time domain is shown in Fig. 2(a). The bit rate of the applied PRBS is $500 \mathrm{Mb} / \mathrm{s}$. The detected signal is captured by a real-time oscilloscope with a $350 \mathrm{MHz}$ bandwidth and a $1 \mathrm{GS} / \mathrm{s}$ sampling rate. As the required sampling rate is only $1 / 4$ of the PRBS rate, the data captured by the oscilloscope is sampled for every eight points in the software to realize an equivalent sampling rate of $125 \mathrm{MHz}$. The downsampled signal is shown in Fig. 2(b). In our experiment, we choose the parameters $N=8192$ and $M=N / 4=2048$. We use the sparse recovery algorithm developed by Figueiredo et al. to reconstruct the original signal from the measurement results [13]. The reconstructed signal in the frequency domain and in the time domain is shown in Figs. 2(c) and 2(d), respectively. The accurate signal reconstruction in both the time and frequency domains is observed from the results. The recovery error is around 0.14 , estimated by $\|\hat{\mathbf{x}}-\mathbf{x}\|_{2}^{2} /\|\mathbf{x}\|_{2}^{2}$ [ $\underline{5}$, where $\mathbf{x}$ and $\hat{\mathbf{x}}$ denote the input signal and the recovered signal, respectively.

According to the experimental results, the recovered signal contains a low-frequency component near dc, which will degrade the signal-to-noise ratio (SNR) and, therefore, the signal recovery performance. To overcome this problem, the technique of balanced detection [ $[$ ] can be applied by replacing MZM2 with a dual output MZM and the complementary outputs pass through a dispersive medium with the same dispersion amount. After photodetection, the difference of the complementary signals, which is expressed as $\sum_{k=0}^{S-1} \alpha x(t-k T) r(t-k T)$, is sent to the digitizer. Computer simulations are performed to test the capture of a multitone signal. In the simulation, the signal to be measured includes frequency components of $5,10,12,16$ and $19 \mathrm{GHz}$ within a bandwidth of $20 \mathrm{GHz}$. The bit rate of the PRBS is set as
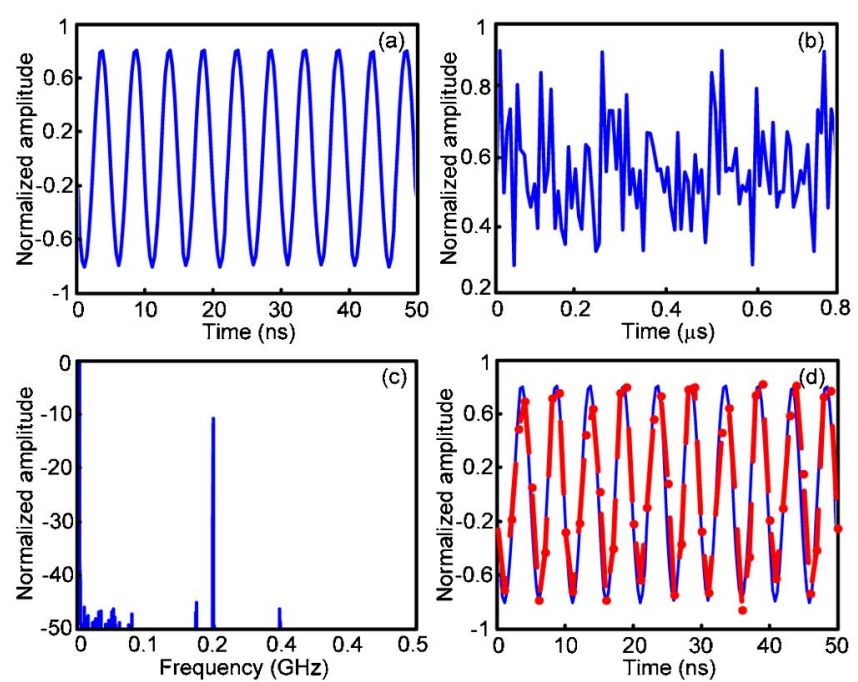

Fig. 2. Experimental results for single-tone signal recovery with compression factor of $4(N=8192)$. (a) Incoming time domain signal, (b) downsampled signal after the process of optical integration, (c) recovered spectrum, and (d) time domain comparison between the original signal (solid) and the reconstructed signal (dashed). 

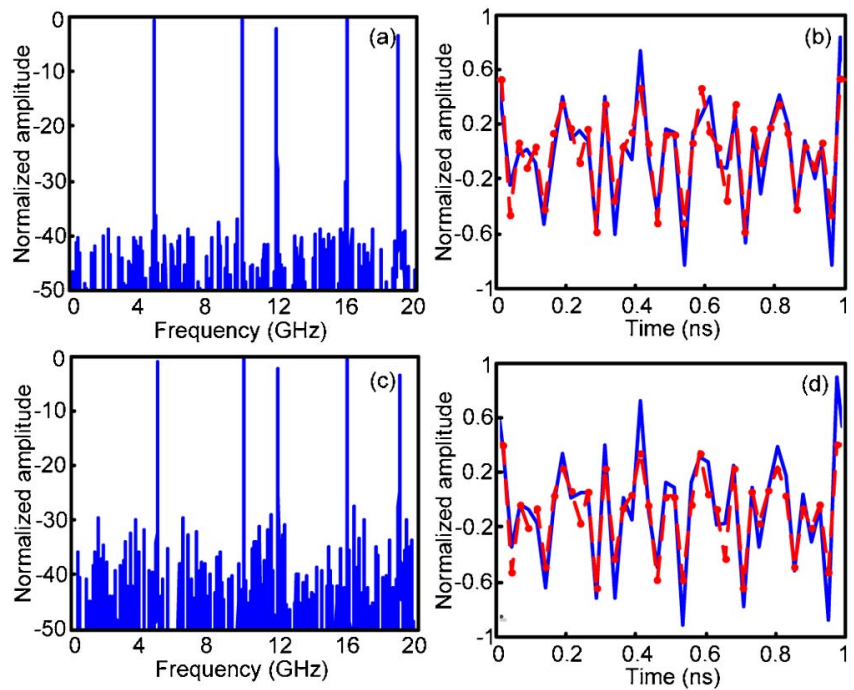

Fig. 3. Signal reconstruction with different compression factors $(N=8192, \mathrm{SNR}=25 \mathrm{~dB})$. (a) and (c) recovered spectra with compression factors of 8 and 16, respectively. (b) and (d) Time domain comparison between the original signal (solid) and the reconstructed signal (dotted) with compression factors of 8 and 16, respectively.

$40 \mathrm{~Gb} / s$, which corresponds to a group delay of $25 \mathrm{ps}$ between the adjacent wavelength channels. The SNR of the input signal is set as $25 \mathrm{~dB}$. The data size $N=$ 8192 is set as in the experiment. Figures $3(\mathrm{a})$ and $3(\mathrm{~b})$ show the recovered spectrum and the time-domain signal when the compression factor is 8 (corresponding to a system with eight wavelengths). In this case, the sampling rate of the digitizer is $40 / 8=5 \mathrm{GS} / \mathrm{s}$. Figures $3(\mathrm{c})$ and $3(\mathrm{~d})$ give the recovered spectrum and time-domain signal when the compression factor is 16 (corresponding to a system with 16 wavelengths). In this case, the sampling rate of the digitizer is $40 / 16=2.5 \mathrm{GS} / \mathrm{s}$. It is seen that the recovery performance degrades with the increase of the compression factor, while, in general, the multitone signal is recovered correctly even under a high compression factor. To evaluate the performance of signal reconstruction, we calculated the recovery error averaged over 100 simulations under different SNRs and compression factors [5]. The results are shown in Figs. 4(a) and 4(b).

In summary, we have proposed a scheme to realize photonic CS with the technique of optical integration. In the system, a multiwavelength optical source and dispersion medium are used to implement the function of delay-and-sum, which is required in CS. The wavelengths should be set properly to ensure that the group delay between adjacent wavelengths matches well with the bit rate of the applied PRBS, which is also the major limitation of the approach. However, compared with the
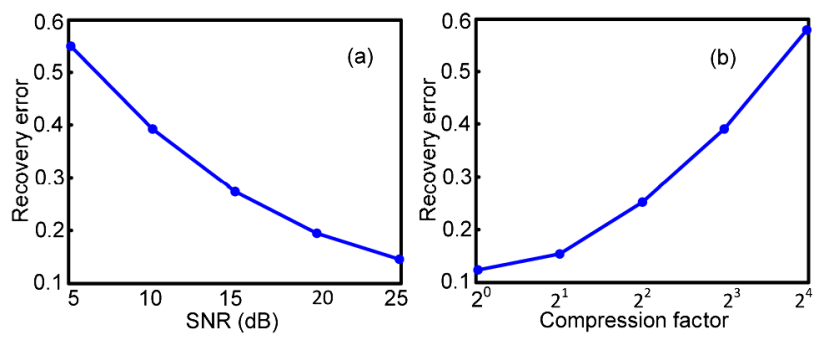

Fig. 4. Averaged recovery error as a function of (a) SNR (compression factor $=4$ ) and (b) compression factor $(\mathrm{SNR}=25 \mathrm{~dB})$.

optical integration scheme in [9], the use of a continuouswave laser source in our approach is much simpler. Single-tone signal recovery in a system with four wavelengths is experimentally demonstrated and multitone signal recovery with more wavelengths is numerically demonstrated to verify the correctness and feasibility of the given method. We believe the demonstrated technique is highly promising for the acquisition of ultrawideband sparse signals.

This work was partially supported by the National Basic Research Program of China (973 Program 2012CB315703), the National Natural Science Foundation of China (NSFC 61177003 and 61171036), and the Program for New Century Excellent Talents in University of China (NCET-10-0723).

\section{References}

1. E. J. Candes and M. B. Wakin, IEEE Signal Process. Mag. 25 (2), 21 (2008).

2. G. C. Valley and G. A. Sefler, Proc. SPIE 7797, 77970F (2010).

3. G. C. Valley, G. A. Sefler, and T. J. Shaw, Opt. Lett. 37, 4675 (2012).

4. J. M. Nichols and F. Bucholtz, Opt. Express 19, 7339 (2011).

5. J. M. Nichols, C. V. McLaughlin, F. Bucholtz, and J. V. Michalowicz, Appl. Opt. 51, 6448 (2012).

6. H. Nan, Y. T. Gu, and H. M. Zhang, IEEE Photon. Technol. Lett. 23, 67 (2011).

7. Y. Liang, M. Chen, H. Chen, C. Lei, P. Li, and S. Xie, Opt. Express 21, 25700 (2013).

8. Y. Chen, H. Chi, T. Jin, S. L. Zheng, X. F. Jin, and X. M. Zhang, J. Lightwave Technol. 31, 3395 (2013).

9. B. T. Bosworth and M. A. Foster, Opt. Lett. 38, 4892 (2013).

10. H. Chi, Y. Mei, Y. Chen, X. M. Zhang, X. M. Zhang, S. L. Zheng, and X. F. Jin, Opt. Lett. 37, 4636 (2012).

11. F. Yin, Y. Gao, Y. Dai, J. Zhang, K. Xu, Z. Zhang, J. Li, and J. Lin, Opt. Lett. 38, 4386 (2013).

12. L. Yan, Y. Dai, K. Xu, J. Wu, Y. Li, Y. Ji, and J. Lin, IEEE Photon. J. 4, 664 (2012).

13. M. A. T. Figueiredo, R. D. Nowak, and S. J. Wright, IEEE J. Sel. Top. Signal Process. 1, 586 (2007). 